\title{
The Grain Growth after Light Cold Rolling in Grain-Oriented Silicon-Iron Sheets*
}

\author{
By Hitoshi Nakae**
}

\begin{abstract}
The grain growth after light rolling below $10 \%$ in grain-oriented silicon-iron sheets containing 3.50 and $1.43 \%$ Si was studied optically and magnetically in ordsr to obtain fundamental data for the establishment of a strain-annealing procedure as a modification of the Goss process. It has been found that, in initially fine-grained sheet specimens, the grain growth increases more prominently at two reduction rates, namely, at 0.5 to $2 \%$ and 6 to $7 \%$. Their resulting preferred orientations are different; in the former, a marked development of the Goss texture occurred, possibly involving no primary recrystallization, while in the latter recrystallization from the Goss texture also occurred.

The recrystalization, from the Goss texture occurred also on initially coarse-grained specimens. It has been found that the optimum point for producing silicon-iron sheets with high permeability and low hysterezis loss is at the first grain growth maximum of $2 \%$ reduction for initially fine-grained sheet specimens.
\end{abstract}

(Received September 4, 1961)

\section{Introduction}

Goss ${ }^{(1)}$ reported first in 1935 a method of producing grain-oriented silicon-iron sheets with grains of a (110) plane parallel to the rolling plane and a $[001]$ direction parallel to the rolling direction, this grain texture being now commonly called the Goss texture. Goss's procedure is to cold-roll purified silicon-iron sheets containing 3 to $3.5 \%$ Si to 30 to $40 \%$ of their original thickness, repeating the same cold rolling again after an intermediate annealing at 850 to $900^{\circ} \mathrm{C}$, and finally to anneal them at about $1100^{\circ} \mathrm{C}$ in pure dry hydrogen.

In 1947, Jackson ${ }^{(2)}$ and, independently, the present author ${ }^{(3)}$ presented a modification of the Goss procedure, in which silicon-iron sheet already cold-rolled to 30 to $40 \%$ of their original thickness and then annealed at 850 to $900^{\circ} \mathrm{C}$, namely, subjected to the first stage of the Goss procedure, was again cold-rolled by a small percentage and annealed at $850^{\circ} \mathrm{C}$ in pure hydrogen. The last stage of our procedure is similar to the so-cold strain-anneal technique for growing single crystals and so the author's procedure is, so to speak, the first stage of the Goss procedure plus the strain-anneal procedure. Our procedure produces a well developed, Goss texture with a large grain size, and it is now used in many steel mills. But little is known as to the behavior of crystal grains during the strain-anneal stage of our procedure, which is the subject of the present study.

\section{Specimens and Experimental Procedure}

The specimens used for the present study are 3.50 and $1.43 \%$ silicon-irons, of which the chemical compositions are shown in Table 1 . The starting materials are $1.6 \mathrm{~mm}$ thick sheets as produced by hot rolling from

** Tsurumi Research Laboratory, Tokyo-Shibaura Electric Co. Ltd., Tokyo, Japan. Presently at the Department of Precision Engineering, Faculty of Engineering, Hokkaido University, Sapporo, Japan.

* Read first at the General Meeting of the Japan Institute of Metals held at Tokyo, Japan, April 1948 and at Akita, Japan, October 1961.

(1) N.P. Goss: T.A.S.M., 23 (1935), 511.

(2): J. M. Jackson: Japan Patent, No. 7504.

(3) Read at the General Meeting of the Japan Institute of Metals held at Tokyo, Japan, April 1948. ingots. These sheets were annealed àt $800^{\circ} \mathrm{C}$, cold rolled to $0.35 \mathrm{~mm}$ in thickness, and then annealed at $900^{\circ} \mathrm{C}$ in pure dry hydrogen, namely, subjected approximately to the first stage of the Goss procedure. In these prepared sheets, the grains are microscopic in size, and

Table 1 Weight percent chemical compositions of the silicon-iron sheet specimens used.

\begin{tabular}{c|c|c|c|c|c}
\hline $\mathrm{Si}$ & $\mathrm{G}$ & $\mathrm{Mn}$ & $\mathrm{P}$ & $\mathrm{S}$ & $\mathrm{Cu}$ \\
\hline 3.50 & 0.005 & 0.16 & 0.013 & 0.023 & 0.30 \\
\hline 1.43 & 0.010 & 0.11 & 0.016 & 0.032 & 0.16 \\
\hline
\end{tabular}

we call them, for brevity, fine-grained sheet specimens in the following. Further, to obtain coarse-grained sheet specimens with the grain size of several $\mathrm{mm}$ to about $20 \mathrm{~mm}$, the fine-grained $3.50 \%$ silicon-iron sheets were cold-rolled $1 \%$ of their original thickness and annealed at $850^{\circ} \mathrm{C}$ in pure dry hydrogen. Then, the fine grained as well as the coarse-grained sheet specimens were cold-rolled 0.5 to $10 \%$ of their original thickness and annealed at $850^{\circ} \mathrm{C}$ in pure dry hydrogen. As mentioned in the introduction, this final procedure will be called, for brevity, the strain-anneal stage of our procedure hereafter. It is to be noted that, in the strain-anneal stage of our procedure, the cold rolling of fine grained $3.50 \%$ silicon-iron sheet specimens were also made in 30,60 and $90^{\circ}$ angle directions to the initial rolling direction.

With these prepared sheet specimens, crystal grains were observed by the naked eye after being macroetched with a $10 \%$ aqueous solution of $\mathrm{HNO}_{3}$ and the magnetic characteristics were measured by a magnetic permeameter. Also, the degree of the development of the Goss texture was determined by a torque magnetometer using discs $2 \mathrm{~cm}$ in diameter cut from the sheet specimens.

III. Experimental Results and Discussions

(1) Experiments on fine-grained sheet specimens

The macrostructures, after being subjected to the 
strain-annealing of fine-grained 3.50 and $1.43 \%$ siliconiron sheet specimens are shown in Photo. 1. Photo. 1 shows that the grain growth is most conspicuous at $2 \%$ reduction for $3.50 \%$ silicon and at $3 \%$ reduction for $1.43 \%$ silicon, respectively, and that the second growth maximum of the grain growth occurs at 6 to $7 \%$ reduction for both silicon contents, the second maximum at $7 \%$ reduction for $1.43 \%$ Si being more significant.

$$
\begin{aligned}
L=-d E / d \theta=-K_{1}(2 \sin 2 \theta+3 \sin 4 \theta) / 8 \\
+K_{2}(\sin 2 \theta-4 \sin 4 \theta-3 \sin 6 \theta) / 64
\end{aligned}
$$

where $E$ is the ferromagnetic anisotropy energy density, $\theta$ is the angle between the direction of magnetization vector and the [001] direction, and $K_{2}$ is the second cubic ferromagnetic anisotropy constant. For the actual comparison, of the measured and theoretical torque curves the first negative maximum, $\mathrm{Lm}_{1}$, and the ratio
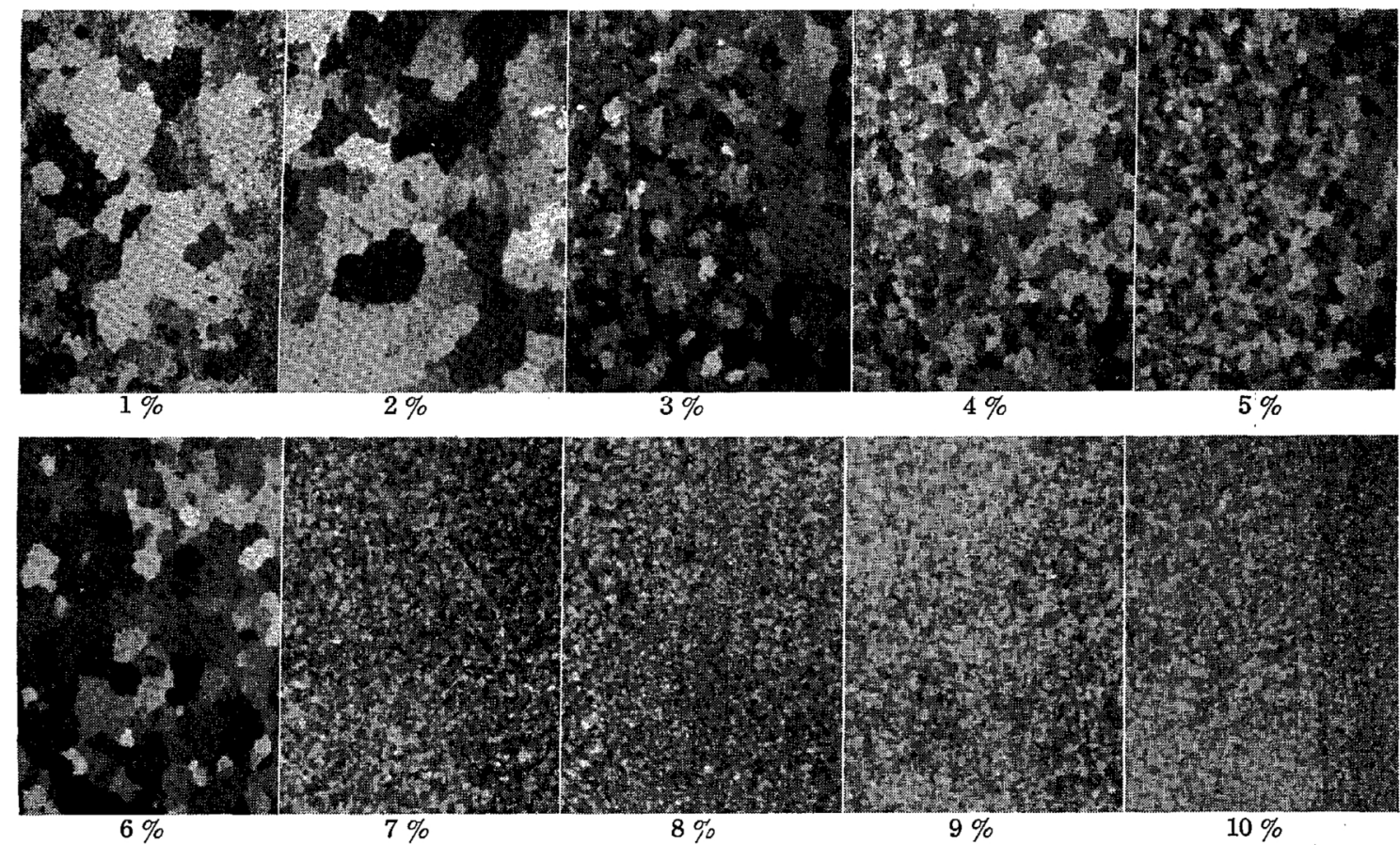

(a) $3.50 \%$ Silicon-Iron

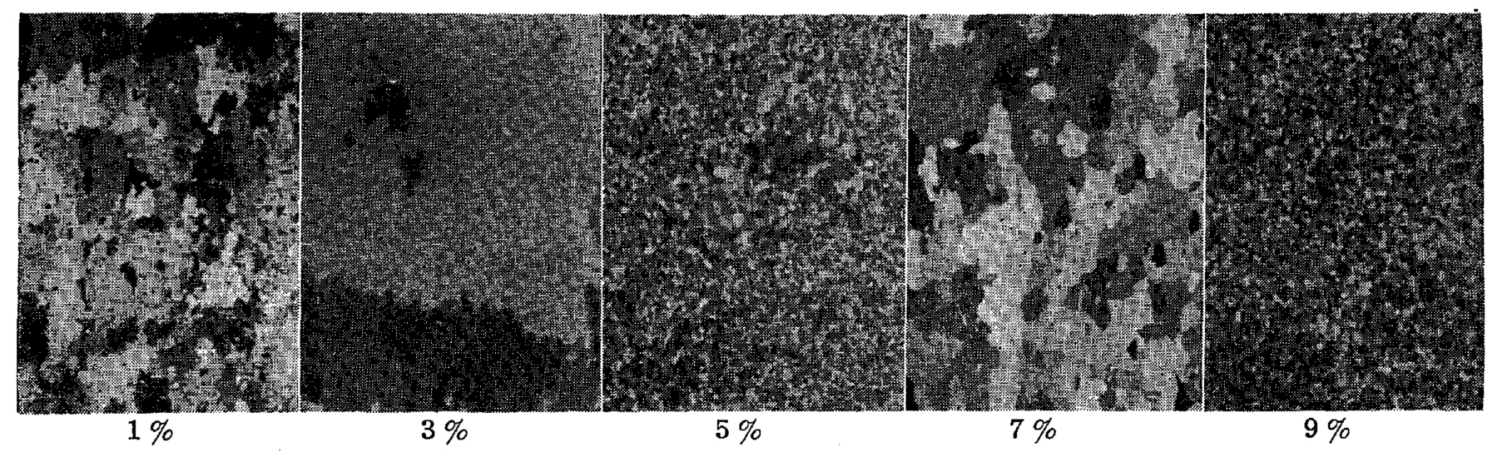

(b) $1.43 \%$ Silicon-Iron

Photo. 1 Effect of the reduction rate adopted in the strain-annealing stage of the author's procedure on the grain size of fine-grained 3.50 and $1.43 \% \mathrm{Si}-\mathrm{Fe}$ sheet specimens. Numbers under photographs indicate the values of the reduction rate. Actual size.

The occurrence of two growth maxima seems to have been found by Stanley(4) with some silicon-iron sheets as simply strain-annealed. It is to be noted that $3.50 \%$ silicon-iron sheet specimens cold-rolled in directions different from the initial rolling direction in the strainannealing stage of the author's procedure also showed quite similar macrostructures.

Next, the degree of development of the Goss texture in the sheet specimens may be determined from a comparison of the measured magnetic torque curves with the theoretical curves for the (110) [001] single crystal with a positive cubic ferromagnetic anisotropy constant, $K_{1}$, which is expressed as;

(4) J. K. Stanley: Trans. AIME, 162 (1952), 116, cited by J, E. Burke et al in Progress in Metal Physics, 3 (1952). 242. of the second positive maximum, $\mathrm{Lm}_{2}$, to $\mathrm{Lm}_{1}$ have an important meaning. Because, for instance, when the texture of the sheet specimen has the (110) plane of grains coinciding with the rolling plane and the [001] direction of grains centering at the two symmetrical directions nearby the rolling direction in the rolling plane, as is commonly seen in grain-oriented silicon-iron sheets, $\mathrm{Lm}_{1}$ is lower than, but $\mathrm{Lm}_{2} / \mathrm{Lm}_{1}$ is the same as, in the case of a single crystal, while the texture has the [001] directions of grains coinciding with the rolling direction and the (110) planes of grains fluctuating about the rolling plane, $\mathrm{Lm}_{1}$ is lower, but $\mathrm{Lm}_{2} / \mathrm{Lm}_{1}$ is higher, than in a single crystal. Fig. 1 shows typical magnetic torque curves, measured after being subjected only to the first stage of the Goss procedure or of the author's procedure and after being subjected to the 
whole of our procedure, of fine-grained $3.50 \%$ siliconiron sheet specimens as well as the theoretical torque. curve of a (110) [001] single crystal of $3.0 \%$ silicon iron for which $\mathrm{Lm}_{1}$ and $\mathrm{Lm}_{2} / \mathrm{Lm}_{1}$ are $16.5 \times 10$ dyne$\mathrm{cm} / \mathrm{cm}^{3}$ and 0.43 . respectively ${ }^{(5)}$.

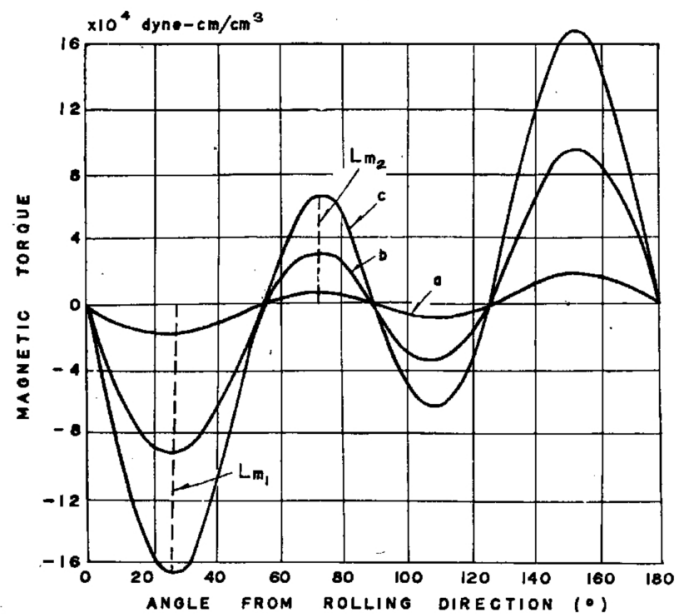

Fig. 1 Typical magnetic torque curves, measured after subjection only to the first stage of the Goss procedure (or of the author's procedure) (a) and after subjection to the author's entire procedure, (b) of fine-grained $3.50 \% \mathrm{Si}-\mathrm{Fe}$ sheet specimens and the theoretical curve of a (110) [001] single crystal of $3.0 \% \mathrm{Si}-\mathrm{Fe}$ with positive $K_{1}(\mathrm{c})$.

In Fig. 2, the measured $\mathrm{Lm}_{1}$ and $\mathrm{Lm}_{2} / \mathrm{Lm}_{1}$ values are plotted against the reduction rate adopted in the strainannealed stage of the author's procedure for fine-grained 3.50 and $1.43 \%$ silicon-iron sheet specimens. It may be seen, from a comparison of Fig. 2 and Photo. 1, that

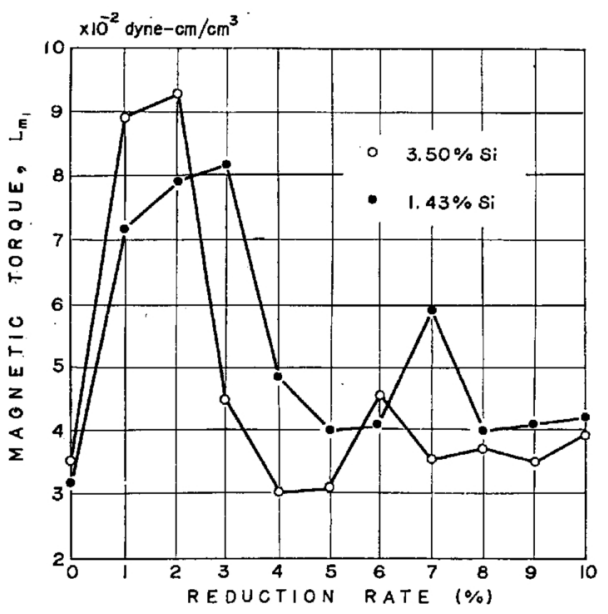

(a) $L_{m_{t}}$

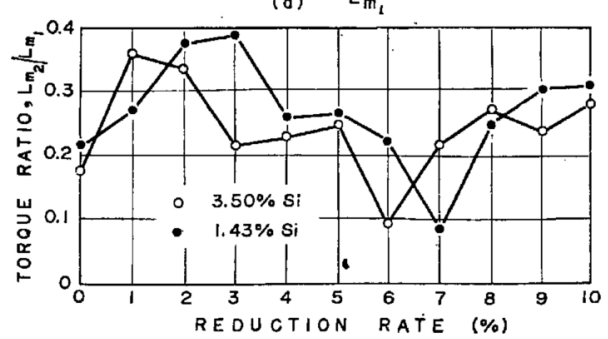

(b) $L_{m_{2}} / L_{m_{1}}$

Fig. 2 Effect of the reduction rate used during the strainanneal stage of the author's procedure on the magnetic torque maximum (negative), $\mathrm{Lm}_{1}$, and the ratio of the first and second magnetic torque maxima, $\mathrm{Lm}_{2} / \mathrm{Lm}_{1}$, for fine grained 3.50 and 1.43 $\% \mathrm{Si}-\mathrm{Fe}$ sheet specimens. the $\mathrm{Lm}_{1}$ value and the degree of grain growth as dependent on the reduction rate adopted in the strainannealing stage of the author's procedure are alike, irrespective of the difference of silicon content. Thus, $\mathrm{Lm}_{1}$ also shows two maxima at the reduction rate where the grain growth is maximum, whereas $\mathrm{Lm}_{2} / \mathrm{Lm}_{1}$ shows a maximum value approximate to the theoretical value 0.43 for a (110) [001] single crystal of $3.0 \%$ silicon iron at the reduction rate percentage where the first growth maximum locates, but it shows a minimum value at a reduction rate where the second growth maximum appears. Those results indicate that the Goss Texture is developed nearly perfectly at the reduction rate percentage where the first grain growth maximum appears, while, at the reduction rate corresponding to the second grain growth maximum, some other texture is also developed besides that of the Goss Texture.

Further, the torque measurements indicate that the initially fine-grained $3.50 \%$ silicon-iron sheet specimens cold-rolled in directions different from the initial rolling direction in the strain-annealing stage of the author's procedure and showing macrostructures quite similar to those of the specimens cold-rolled in the initial rolling direction, as mentioned above, have a Goss texture which is already developed in the first stage of the Goss procedure, or of the author's procedure and has a distribution of grain orientation relative to the initial rolling direction, similar to that in the specimens made by cold rolling in the initial rolling direction.

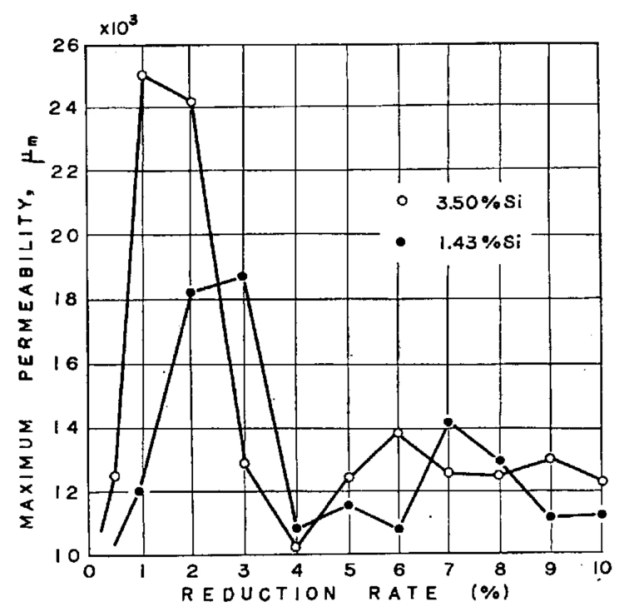

(a) $\mu_{\max }$

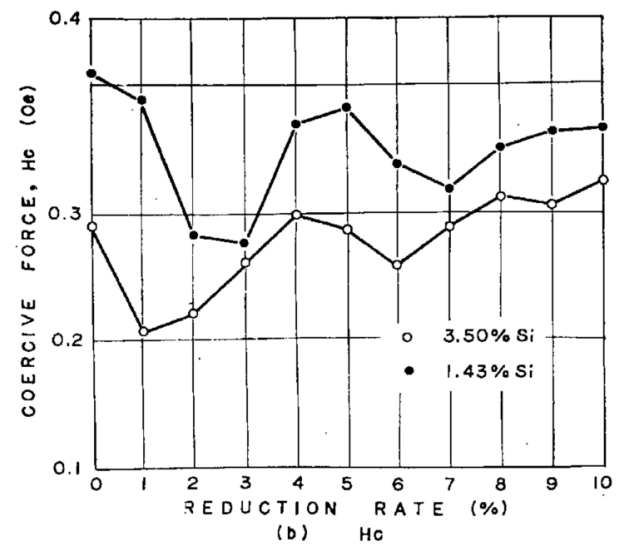

Fig. 3 Effect of the reduction rate used during the strainanneal procedure on maximum permeability, $\mu_{\max }$, and coercive force, $\mathrm{Hc}$, for fine-grained 3.50 and $1.43 \% \mathrm{Si}-\mathrm{Fe}$ sheet specimens. 
It seems that Stanley(4) attributes the two growth maxima observed with simply strain-annealed silicon iron sheets to the small nucleation activities of the primary recrystallization, but it may be concluded, from the above-mentioned macrostructural observations and magnetic torque measurements, that crystals developed at the first growth maximum in the initially fine-grained Goss-texture specimens have been grown from the Goss crystals with a small orientation fluctuation relative to the (110) [001] single crystal, possibly involving no primary recrystallization, and a crystal developed at the second growth maximum are a mixture of the Goss crystals and crystals belonging to certain textures recrystallized from the Goss texture.

Finally, the measured values of the maximum permeability, $\mu_{\max }$, and of the coercive force, $\mathrm{H}_{\mathrm{C}}$, are plotted against the reduction rates adopted in the strain-annealing stage of our procedure in Fig. 3. It may be seen from this figure that $\mu_{\max }$ shows two maxima, while $\mathrm{H}_{C}$ shows two minima, just corresponding to the two maxima observed with the grain growth (Photo. 1) and with $\mathrm{Lm}_{1}$ (Fig. 2). Moreover, the magnetic hysteresis loops are highly rectangular compared with those of silicon-iron
$0.35 \mathrm{~mm}$ thick, with various grain sizes below $3 \mathrm{~mm}$. It has been found that the first grain growth maximum occurring at reduction rates between 2 and $3 \%$ used for the strain-annealing stage of the author's procedure in fine-grained 3.50 and $1.43 \%$ silicon-iron sheet specimens tends to disappear when the diameter of the grain is about $3 \mathrm{~mm}$. This critical grain size is actually affected by the following conditions of the specimens before testing, thickness of specimen, amount of impurities, rolling conditions and texture of the initial specimen.

Then, detailed strain-annealing experiments were carried out using coarse-grained $3.50 \%$ silicon-iron sheet specimens with a grain size much larger than 3 $\mathrm{mm}$, which were prepared by $1 \%$ cold reduction and subsequent annealing at $850^{\circ} \mathrm{C}$ in dry hydrogen. It was observed that, below $2 \%$ cold reduction and at the annealing temperature of $850^{\circ} \mathrm{C}$, the grain growth maximum did not appear and the texture was not changed very much, but, over $3 \%$ reduction and at annealing temperatures higher than $850^{\circ} \mathrm{C}$, new recrystallization occurred in the Goss crystals, as seen in Photo. 2, which show the grain structure before and after the strain

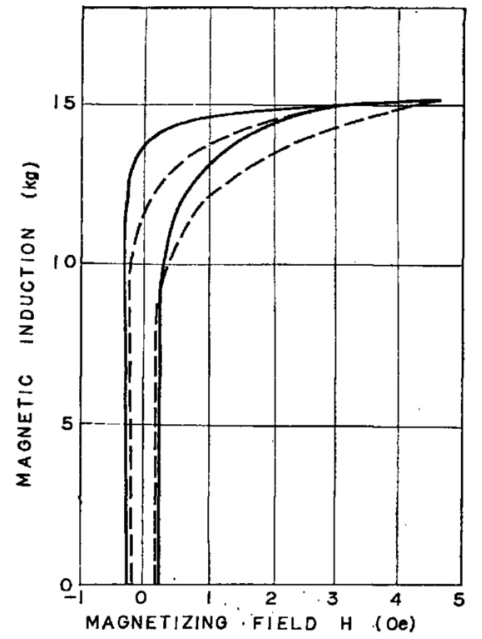

Fig. 4 Hysteresis loops of a $3.50 \% \mathrm{Si}-\mathrm{Fe}$ sheet specimen prepared from a fine-grained sheet specimen by our procedure (the reduction rate adopted in the strain anneal stage of our procedure is $2 \%$ ) (solid line) as compared to the Gross procedure (broken line).

sheets with nearly the same degree of development of the Goss texture as prepared by the Goss procedure, as shown, for example, in Fig. 4.

Thus, based on the above-mentioned experimental results, we could obtain highly grain-oriented siliconiron sheets with high permeability and low iron loss when using a low reduction rate of $2 \%$ where the first grain growth maximum appeared.

\section{(2) Experiments on coarse-grained sheet specimens}

First the influence of the grain size on the basic materials which were subjected to the first stage of the Goss procedure (or of the author's procedure) on the grain growth obtained by the subsequents train-annealing stage of the author's procedure was studied using coldrolled and hot-rolled 1.2 to $3.5 \%$ silicon-iron sheets,

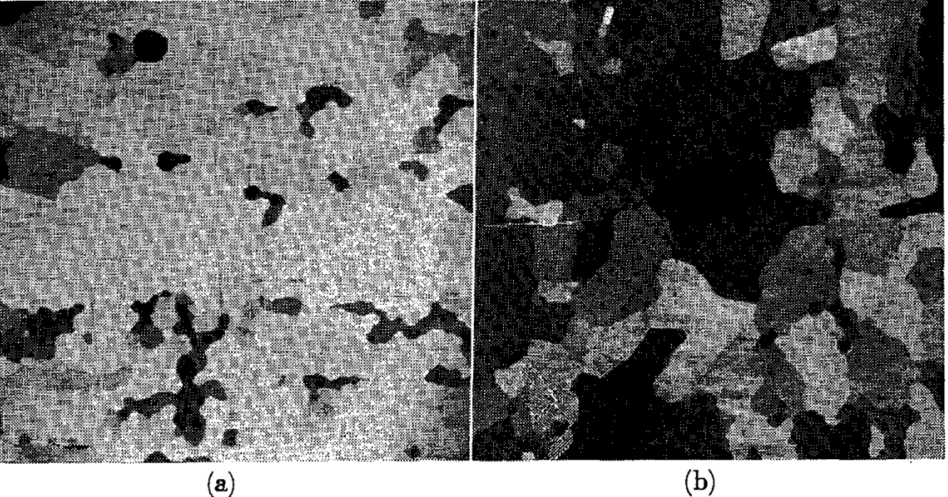

Photo. 2 Effect of the strain anneal $\left(3 \%\right.$ cold reduction and $850^{\circ} \mathrm{C}$ annealing) on coarse-grained $3.50 \% \mathrm{Si}-\mathrm{Fe}$ sheet specimens. Photographs show the grain structures in the same region of the specimen before (a) and after (b) the strain anneal.

annealing of $3 \%$ cold reduction and annealing at $850^{\circ}$ C. These photographs were taken of a specimen etched slightly with a $10 \%$ aqueous solution of $\mathrm{HNO}_{3}$ using an optical apparatus similar to that described by Assmus et al. ${ }^{(6)}$. in which the directions of the incident light beam and of the observation direction were the same and

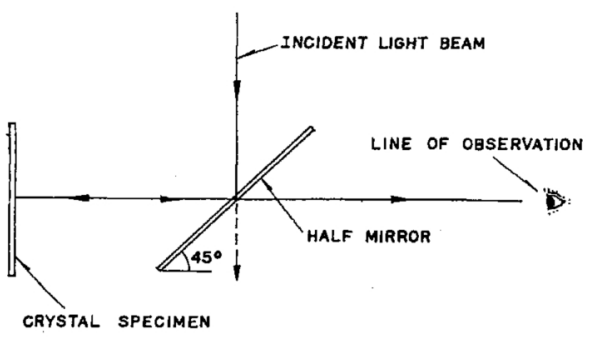

Fig. 5 Optical apparatus used for the observation of the textures of sheet specimens.

perpendicular to the specimen surface, as shown schematically in Fig. 5. We can see from these photographs that the Goss crystals showing a high-degree reflection

(6) F. Assmus, K. Detert and G. Ibe: Z. Metallkde., 48 (1957), 345. 
occupy a large portion of the specimen before the strain annealing, but, after this procedure, they are markedly decreased while the recrystallized dark regions increased.

The orientations of crystals in the recrystallized regions were found to be qualitatively similar to those of crystals found in 6 to $7 \%$ strain-annealed finegrained specimens. Thus, the measured $\mathrm{Lm}_{1}$ and $\mathrm{Lm}_{2} /$ $\mathrm{Lm}_{1}$ values before and after the strain-annealing procedure are shown in Table 2, which indicates that both $\mathrm{Lm}_{1}$, and $\mathrm{Lm}_{2} / \mathrm{Lm}_{1}$, decrease and approach values corresponding to the second grain growth maximum observed with initially fine-grained sheet specimens (Fig. 2).

Table 2 A few examples of $\mathrm{Lm}_{1}$ and $\mathrm{Lm}_{2} / \mathrm{Lm}_{1}$ values of initially coarse-grained $3.30 \% \mathrm{Si}-\mathrm{Fe}$ sheet specimens before and after the strain-anmealing procedure.

\begin{tabular}{c|c|c|c|c}
\hline \hline \multirow{2}{*}{ Specimen, No. } & \multicolumn{2}{|c|}{$\operatorname{Lm}_{1}(10$ dyne-cm/cm) } & \multicolumn{2}{|c}{ Lm. $_{2} / \mathrm{Lm}_{1}$} \\
\cline { 2 - 5 } & Before & After & Before & Af $\omega_{-}$ \\
\hline 1 & 10.3 & 7.6 & 0.38 & 0.12 \\
2 & 14.4 & 9.3 & 0.41 & 0.11 \\
3 & 9.1 & 7.1 & 0.34 & 0.16 \\
\hline
\end{tabular}

These facts may be summarized as follows:

(i) The recrystallization takes place in Goss erystals in the second grain growth maximum of 6 to $7 \%$ coldrolled fine-grained sheet specimens as well as in more
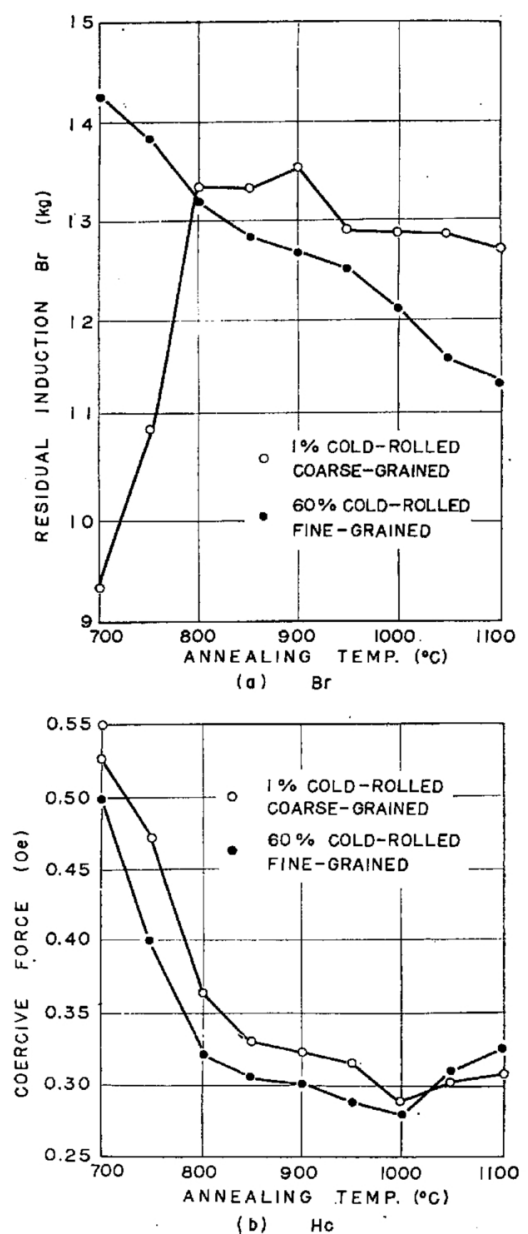

Fig. 6 Effect of the annealing temperature on the residual induction, $\mathrm{Br}$, and coercive force, $\mathrm{Hc}$, of strain-annealed coarse-grained $3.50 \% \mathrm{Si}-\mathrm{Fe}$ sheet specimens as compared with $3.50 \% \mathrm{Si}-\mathrm{Fe}$ sheet specimens prepared by the Goss procedure (60\% cold rolled). than $3 \%$ cold-rolled coarse-grained sheet specimens.

(ii) The difference in the above reduction rate may be attributed to the fact that in fine grained sheet specimens, the first grain growth tendency shifts the primary recrystallization to a 6 to $7 \%$ reduction rate, revealing the second grain growth maximum.

It was noticed in (1) that the hysteresis loops of the specimens produced by our strain-annealing procedure were highly rectangular. In Figs. 6 and 7, the values of the residual induction $\mathrm{Br}$, and coercive force $\mathrm{Hc}$, measured on coarse-grained sheet specimens are functions of the reduction rates and of the annealing temperature adopted in the strain-annealing stage of our procedure.

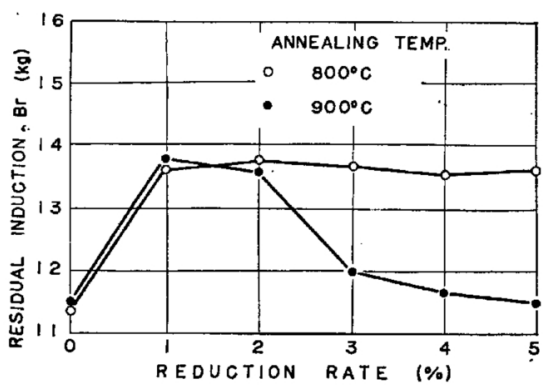

Fig. 7 Effect of the reduction rate on the residual induction of the strain-annealed coarsegrained $3.50 \% \mathrm{Si}-\mathrm{Fe}$ sheet specimens.

Fig. 6 (a) shows the residual induction $\mathrm{Br}$ for the maximum induction of 15 kilo-Gauss as dependent on the annealing temperature as compared with that of the Goss processed specimens. In coarse grained specimens, $\mathrm{Br}$ increases rapidly with increasing annealing temperature, passes a maximum value and then decreases to some extent whereas that of the Goss processed specimen decreases uniformly with rising annealing temperature. On the other hand, as seen from Fig. 6(b), Hc decreases with increasing annealing temperature for both specimens. The decrease of $\mathrm{Br}$ from a maximum value in coarsegrained specimens (Fig. 7) corresponds to the appearance of recrystallization.

\section{Summary and Conclusions}

The grain growth after being light cold rolled at rates of 3.50 and $1.43 \%$ of silicon-iron sheets with Goss texture was investigated, using both fine-grained and coarse-grained sheet specimens.

Sheet specimens prepared as above, were macro-etched with a $10 \%$ aqueous solution of $\mathrm{HNO}_{3}$ and visually inspected for crystal grains and the magnetic characteristics were measured by a magnetic permeameter. Also the degree of the development of the Goss texture was determined by a torque magnetometer.

In originally fine-grained sheet specimens, two maxima. of the grain growth were found in rolling reduction ranges between 0.5 to $2 \%$ and from 6 to $7 \%$, respectively.

It may be concluded, from the above facts, that at the first grain growth maximum, the resultant crystals. have been grown from the Goss crystals with small orientation fluctuations in the specimens, and possibly the primary recrystallization has not occurred and that, at the second growth maximum, the resultant cystals have been developed by primary recrystallization from the Goss crystals. 
In originally coarse-grained specimens, the first grain growth maximum was not found and it may be considered that, at about $3 \%$ reduction, the primary recrystallization occurs in the Goss crystals and the resulting textures corresponds to those found in the second grain growth maximum in initially fine grained specimens.

It may be concluded from the observed magnetic characteristics, that the optimum point to obtain highly oriented silicon-iron sheets with high permeability and low hysteresis loss is at the first grain growth maximum found with initially fine-grained sheet specimens.

Rectangular shaped hysteresis loops were found with initially fine-grained as well as with initially coarsegrained sheet specimens subjected to the strain-annealing procedure.

\section{Acknowledgement}

The author is very grateful for the helpful advice and encouragement of Dr. S. Kaya, President of the University of Tokyo and Dr. M. Yamamoto, Professor of Tohoku University. The present investigation was partially supported by Scientific Research Funds provided by the Ministry of Education for which the author wishes to express his deep appreciation. 